\title{
High Precision Positioning Control for Table Drive System using PID Controller with Nonlinear Friction Compensator
}

\author{
Kazuhiro TSURUTA ${ }^{1}$, Kazuya SATO ${ }^{2}$, Takashi FUJIMOTO ${ }^{1}$, Nobuhiro USHIMI ${ }^{1}$ \\ ${ }^{1}$ Depertment of Engineering, Kyushu Sangyo University, Japan, tsuruta@ip.kyusan-u.ac.jp \\ ${ }^{2}$ Depertment of Science and Engineering, Saga University, Japan, sato@me.saga-u.ac.jp
}

\begin{abstract}
:
In the machine tool industry and the semiconductor manufacture fields, the necessity of high precision and fast response for NC machines is rapidly spreading. In general, it is well known that a positioning control system contains nonlinear friction force, it may cause a serious disadvantage on control performances such as tracking errors, limit cycles and undesired stick-slip motion at very low motion velocity. In order to achieve the high level control performance, the frictional effects have to be removed. In this paper, we proposed a novel PID control method which is based on the idea of sliding mode control for nonlinear friction compensation in a table drive system. Experimental results are given to show the effectiveness of our proposed PID control method compared with the conventional PID method. Especially, the tracking error of our proposed PID control was reduced by more than $2 / 3$ compared to the case of the conventional PID control at the end of a stroke.
\end{abstract}

Keywords: Nonlinear control, Friction compensation, PID control, Sliding mode control method

\section{Introduction}

In the machine tool industry and the semiconductor manufacture fields, the necessity of high precision and fast response for $\mathrm{NC}$ machines is rapidly spreading. In general, it is well known that a positioning control system contains nonlinear friction force, it may cause a serious disadvantage on control performances such as tracking errors, limit cycles and undesired stick-slip motion at very low motion speed. In order to achieve the high level of performance, frictional effects have to be removed. Various control method have been proposed to compensate these behaviors. In the previous researches, there have been focused on the more accurate formulation and parameter identification of friction models[1,2]. Also, they have focused on the compensation algorithms for the friction, e.g., the feed forward compensation, the state feedback regulation, the state observer-based method, soft computing algorithms, and so forth. However, friction is a natural phenomenon that is quite hard to model description, and is not yet completely understood. In applications, the identification of friction parameters is usually a problem because it depends on the velocity, position, temperature and lubrication.

In this paper, we propose a novel PID control method which is based on the idea of sliding mode control for nonlinear friction compensation in table drive systems. The reason why we applied an idea of sliding mode control is that the friction parameters need not be identified. Our proposed PID control method can be regarded as one of the nonlinear control methods. Furthermore, stability is guaranteed to calculate friction compensation force using Lyapunov's direct method. We evaluate our proposed PID control and conventional PID control at low speed in table drive system. The deference between proposed PID control method and conventional PID control method is whether the controller with the compensator or without the compensator. Experimental results with and without compensation are compared to validate the effectiveness of our proposed PID control method.

\section{Nonlinear Frictional Behavior}

Figure 1 shows the photograph of one axis slider and the configuration of an experimental depicts in Figure 2. The experimental system are consisted as : (i) a one-axis stage mechanism consisting of an AC linear coreless motor which has no cogging and no attraction magnet force, (ii) a rolling guide mechanism, (iii) a position-sensor (1pulse=10nm), (iv) two current amplifiers, and (v) a personal computer with the controller, a D/A board and a counter board. Figure 3 shows a block diagram of the proposed control system, where $\lambda_{1}, \lambda_{2}$, and $k$ are design parameters for PID controller, $T_{c}$ is disturbance compensation force,

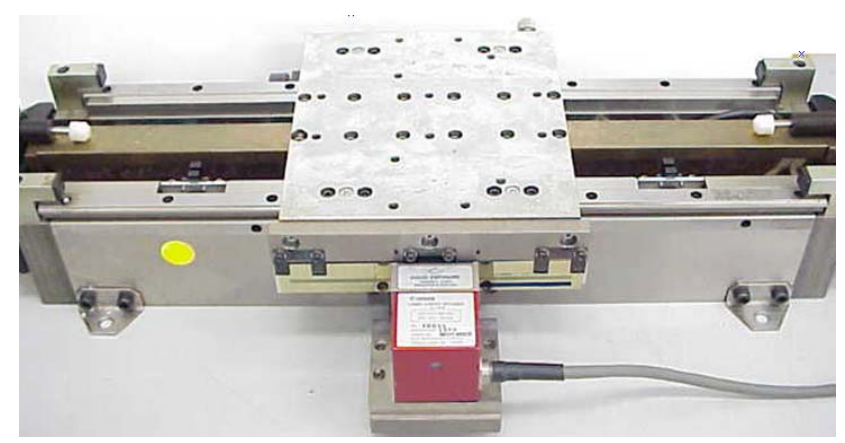

Figure 1: The photograph of one axis slider. 


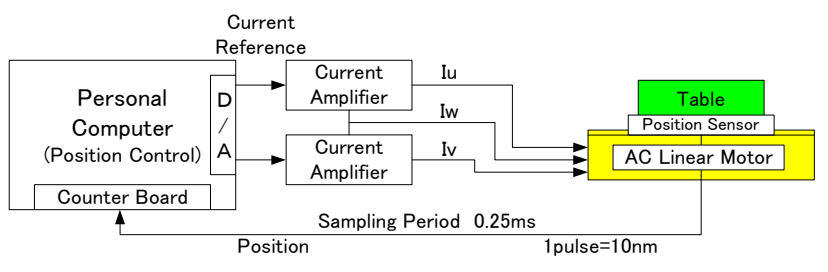

Figure 2: The experimental control system.

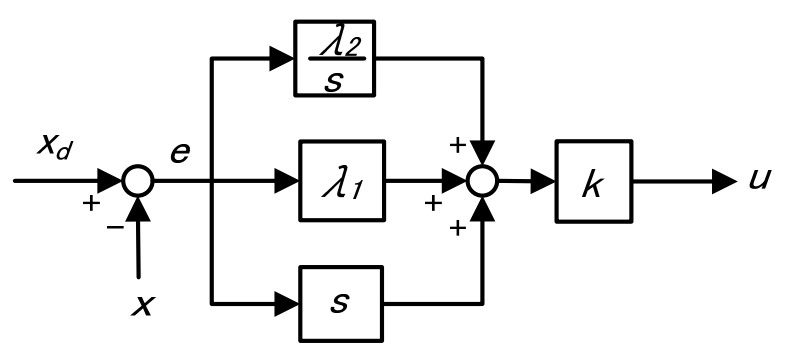

Figure 3: The block diagram of PID control method.

$$
\begin{aligned}
& u=k\left(\dot{e}+\lambda_{1} e+\lambda_{2} \frac{e}{s}\right) \\
& e=x_{d}-x
\end{aligned}
$$

where 's' means Laplace transfer operator. The output of the PID controller can be described as equation (1) and each design parameters are determined by using an appropriate method. Equation (2) shows the tracking error signal where $x_{d}$ is reference signal and $x$ is displacement of the table.

In a practical application, high precision positioning at a low velocity is required, but in general, it is well known that the conventional control methods can not accomplish such a requirement. Moreover, the tracking error becomes large at the end of a stroke because of the effect of a friction force. In the previous researches, a friction force can be regarded as a static function of velocity in spite of its complicated phenomenon. Experiments have shown that there is a deflection or relative movement in the pre-sliding region, indicating that the relationship between the deflection and the applied force resembles a non-linear spring with a hysteretic behavior $[3,4]$. Thus, the present study focuses on the non-linear behavior at the end of a stroke during changes in velocity as shown in Figure 4. In Fig. 4, the signals of (1), (4) and (7) are velocity references, the signals of (2),(5) and (8) are velocity responses, the signals of (3),(6) and (9) are output forces with constant acceleration-deceleration profiles of $10 \mathrm{~mm} / \mathrm{s}, 5.0 \mathrm{~mm} / \mathrm{s}$, and $2.5 \mathrm{~mm} / \mathrm{s}$, respectively. The forces in the actual experiment are calculated values and not the values mechanically measured. It seems that the tracking errors of velocity are almost zero. From Fig. 4, it is seen that the output forces are different during constant velocity and the force of $2.5 \mathrm{~mm} / \mathrm{s}$ is the largest in all cases. The moving force generally needs a big one where velocity is large. The reason is influence of viscous friction. When the velocities are decreasing, output forces have not

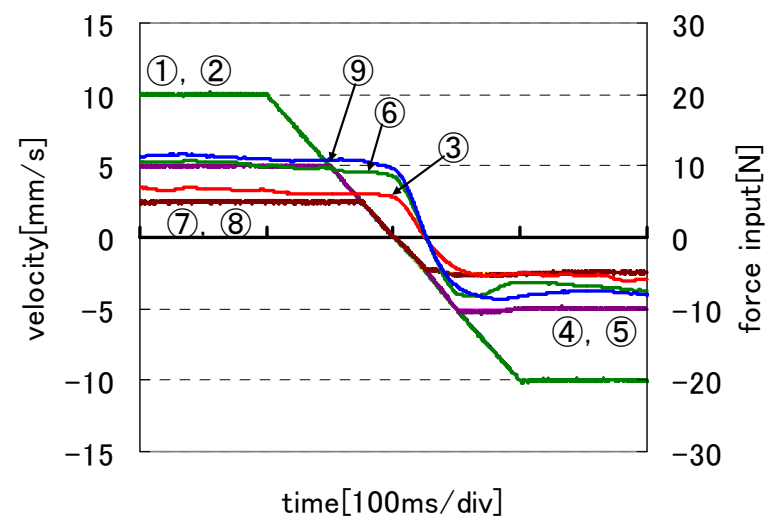

Figure 4: The table motion at the end of a stroke.

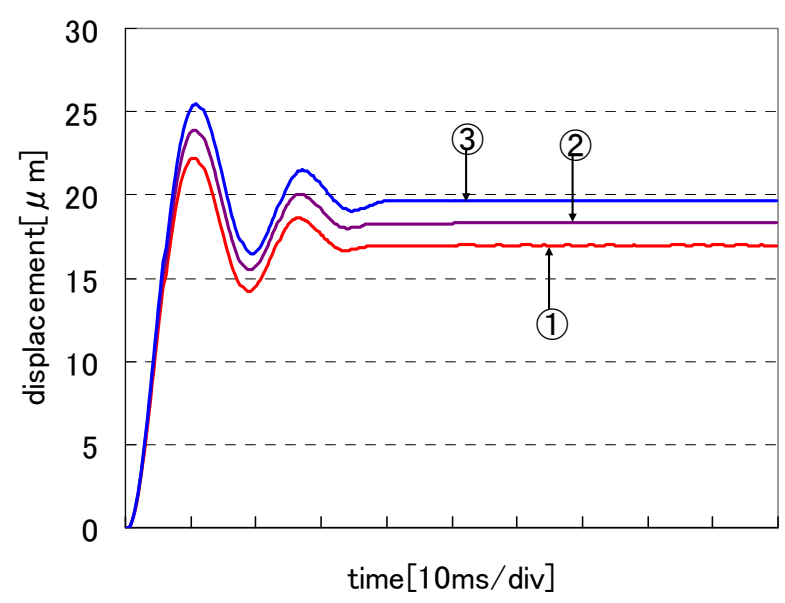

Figure 5: The spring-like behavior.

decreased and when the velocities are increasing, output forces have not increased. Further, when the output forces are set to zero, the spring- like behavior occurs at the end of a stroke, as shown in Fig.5. In Fig.5, the signals of (1), (2) and (3) are the displacement, the command velocities which are $10 \mathrm{~mm} / \mathrm{s}, 5.0 \mathrm{~mm} / \mathrm{s}$, and $2.5 \mathrm{~mm} / \mathrm{s}$, respectively. At values of low command velocities, the spring-like behaviors produce large displacement. The displacement, which exceeds $15 \mu \mathrm{m}$ can negatively influence precision point to point control. The frequency of vibration was observed to be $40 \mathrm{~Hz}$. The spring-like characteristic behavior is thought to be due to the elastic deformation between balls and rails in the ball guide-way [5-7]. Thus, friction is a natural phenomenon that is quite hard to model description by on-line identification, and is not yet completely understood.

\section{Proposed PID control method}

The positioning mechanism is modeled as follows,

$$
M \ddot{x}+D \dot{x}+F=u
$$

where $M$ is mass, $D$ is viscous friction coefficient, $F$ is constant disturbance force, $x$ is displacement and $u$ is force input. The control object is to design a control input $u$ to track a given input reference. In this paper, we will design a PID controller with nonlinear compensator 
for high accuracy, fast response with small overshoot.

First of all, taking second derivative of both sides for equation (2) and substitute it into equation (1), then we have

$$
\begin{aligned}
& M\left(\ddot{x}_{d}-\ddot{e}\right)+D \dot{x}+F=u \\
& M \ddot{e}=-u+M \ddot{x}_{d}+D \dot{x}+F
\end{aligned}
$$

Now, we define a new signal as

$$
r=\dot{e}+\lambda_{1} e+\lambda_{2} \frac{e}{s}
$$

Take time derivative of both sides for Equation (6) and multiply $\mathrm{M}$ to both sides, then we have

$$
\begin{aligned}
& M \dot{r}=M \ddot{e}+M \lambda_{1} \dot{e}+M \lambda_{2} e \\
& =-u+M \ddot{x}_{d}+D \dot{x}+F+M \lambda_{1} \dot{e}+M \lambda_{2} e
\end{aligned}
$$

Besides, we define the augmented signal as equation (8)

$$
\dot{x}_{r}=\dot{x}_{d}+\lambda_{1} e+\lambda_{2} \frac{e}{s}
$$

Then, we can rewrite equation (7) as

$$
M \dot{r}=-u+M \ddot{x}_{r}+D \dot{x}+F
$$

Let the control input $u$ describe as equation (10), where $T_{c}$ will be given later.

$$
u=k r+T_{c}
$$

Substitute equation (10) into (9), we have

$$
M \dot{r}=-k r-T_{c}+M \ddot{x}_{r}+D \dot{x}+F
$$

Consider the Lyapunov function candidate for the closed-loop system as

$$
V=\frac{1}{2} M r^{2}
$$

It's time derivative and using (11) and (12), then we have

$$
\dot{V}=M r \dot{r}=-k r^{2}+r\left(M \ddot{x}_{r}+D \dot{x}+F-T_{c}\right)
$$

To achieve a negative $\dot{V}$, the update low is chosen as

$$
r\left(M \ddot{x}_{r}+D \dot{x}+F-T_{c}\right) \leq 0
$$

with above choice, we obtain

$$
T_{c}=\operatorname{sign}(r)\left(M_{\max }\left|\ddot{x}_{r}\right|+D_{\max }|\dot{x}|+F_{\max }\right)
$$

where $M_{\max }, D_{\max }, F_{\max }$ are maximum values which are predetermined known values. However, chattering phenomena may occur, because equation (15) contains the sign function of $r$ signal. To overcome this problem, we introduce an approximation function of sign function to avoid the chattering as follow

$$
u=k r+\frac{r}{\delta+|r|}\left(M_{\max }\left|\ddot{x}_{r}\right|+D_{\max }|\dot{x}|+F_{\text {max }}\right)
$$

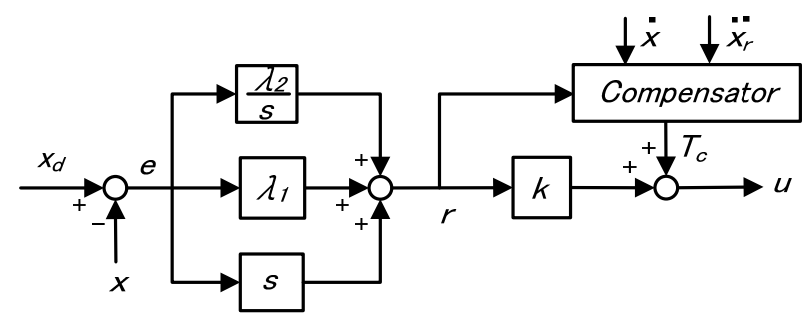

Figure 6: The block diagram of the proposed method.

where $\delta$ is chattering reject parameter. Consequently, time derivative of equation (12) is always negative by choosing sufficient large value of $M_{\max }, D_{\max }, F_{\max }$. Figure 6 shows the block diagram of the proposed nonlinear compensation controller. The second term of right hand side of equation (16) is output of compensator which is shown in Fig. 6.

\section{Experimental results}

To show the effectiveness of the proposed method, experiments were carried out. Digital implementation was assumed in experimental setup. The sampling time of experiments was $0.25 \mathrm{~ms}$. Parameters of PID controller was chosen as $\lambda_{1}=125[1 / \mathrm{s}], \lambda_{2}=5208[1 / \mathrm{s}], \mathrm{k}=62.5[1 / \mathrm{s}]$. The parameters of proposed method was chosen as same

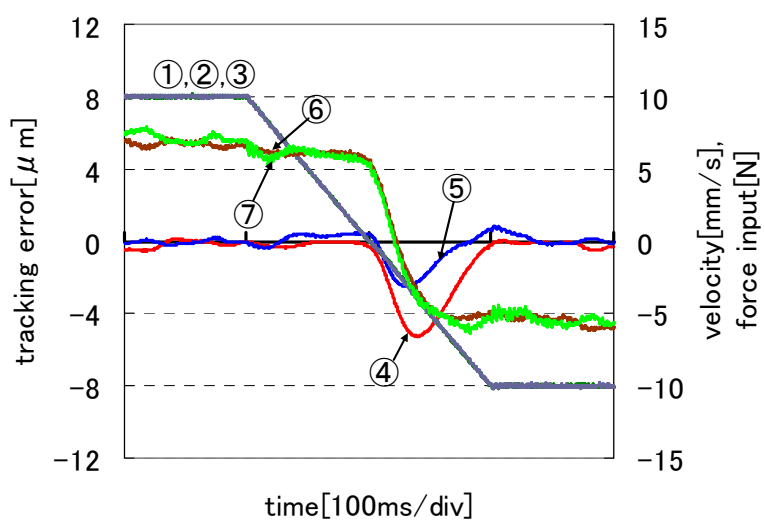

Figure 7: The comparison results of tracking errors in the case of state velocity are $10 \mathrm{~mm} / \mathrm{s}$.

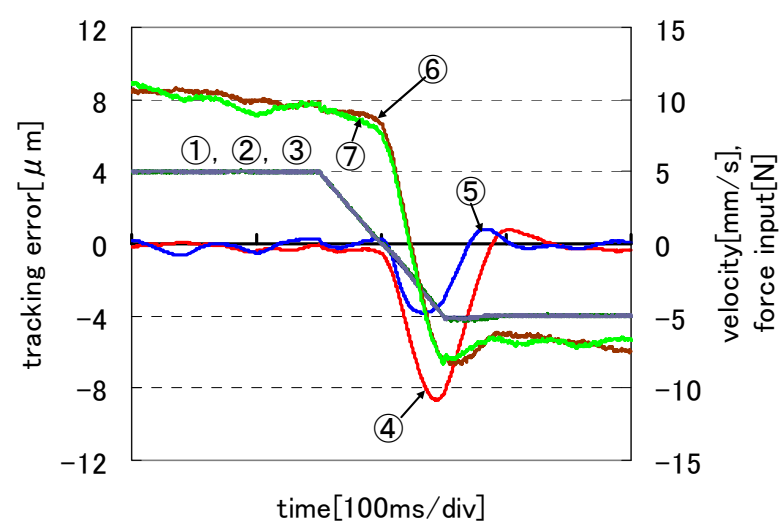

Figure 8: The comparison results of tracking errors in the case of state velocity are $5 \mathrm{~mm} / \mathrm{s}$. 


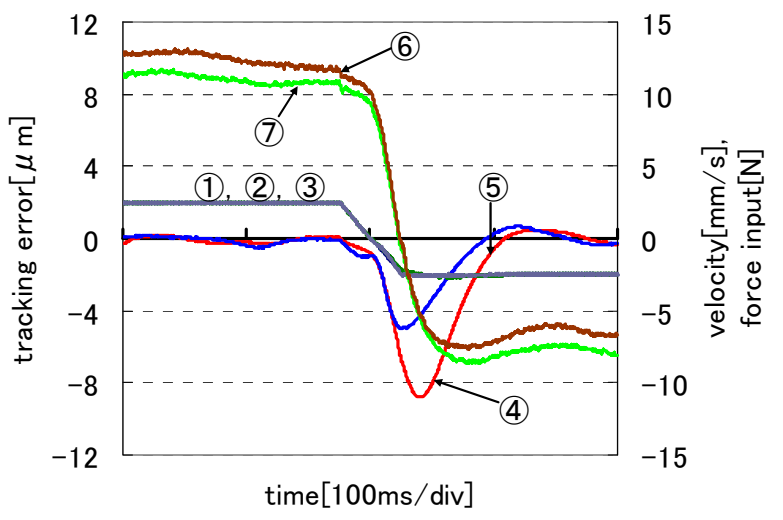

Figure9: The comparison results of tracking errors in the case of state velocity are $2.5 \mathrm{~mm} / \mathrm{s}$.

Table 1: The comparison results of tracking errors at the end of stroke.

\begin{tabular}{|c|c|c|c|}
\hline $\begin{array}{c}\text { Moving } \\
\text { velocity }\end{array}$ & $10 \mathrm{~mm} / \mathrm{s}$ & $5 \mathrm{~mm} / \mathrm{s}$ & $2.5 \mathrm{~mm} / \mathrm{s}$ \\
\hline $\begin{array}{c}\text { Without } \\
\text { compensation }\end{array}$ & $-5.2 \mu \mathrm{m}$ & $-8.6 \mu \mathrm{m}$ & $-8.8 \mu \mathrm{m}$ \\
\hline $\begin{array}{c}\text { With } \\
\text { compensation }\end{array}$ & $-2.8 \mu \mathrm{m}$ & $-3.8 \mu \mathrm{m}$ & $-4.9 \mu \mathrm{m}$ \\
\hline
\end{tabular}

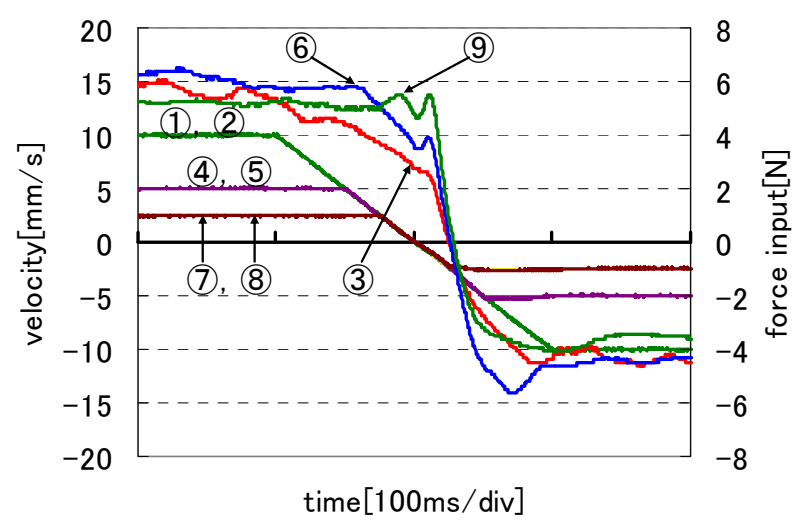

Figure 10: The compensate force inputs among three cases of constant velocity.

value of PID controller and was chosen as $\delta=0.5$. The parameters of $M_{\max }, D_{\max }, F_{\max }$ are set $M_{n} * 5, D_{n} * 5, F_{n} * 5$, respectively. $M_{n}, D_{n}, F_{n}$ are nominal values of the table which are previously obtained. To evaluate the tracking errors at the end of stroke, we used three kind of moving velocities. Figs.7,8,9 show the comparison results of tracking errors in the case of state velocity are $10 \mathrm{~mm} / \mathrm{s}$, $5 \mathrm{~mm} / \mathrm{s}, 2.5 \mathrm{~mm} / \mathrm{s}$, respectively. In Figs.7,8,9, (1) is the velocity reference,(2) is the velocity response without compensation, (3) is the same one with compensation, (4) is the tracking error without compensation, (5) is the same one with compensation, (6) is the force output without compensation, (7) is the same one with compensation, respectively. It is obvious that the tracking errors of the case with compensation are reduced by more than $2 / 3$ compared to the case of without compensation at the end of a stroke. Table 1 shows the tracking errors at the end of stroke. The errors are greatly reduced by our proposed compensation method. Thus, the proposed method is judged to have better performance accuracy. Figure 10 shows the compensate force inputs among three cases of constant velocity. In Fig. 10, the signals of (1), (4) and (7)are velocity references, the signals of (2), (5) and (8) are velocity responses, the signals of (3), (6) and (9) are compensate forces of $10 \mathrm{~mm} / \mathrm{s}, 5.0 \mathrm{~mm} / \mathrm{s}$, and $2.5 \mathrm{~mm} / \mathrm{s}$, respectively. It is clear that the compensation forces are similar to the nonlinear behaviors of Stribeck effect at the end of a stroke.

\section{Conclusion}

In this paper, we proposed a novel PID control method which is based on the idea of sliding mode control for nonlinear friction compensation in table drive systems. Experimental results were given to show the effectiveness of our proposed PID control method compared with the conventional PID method. Especially, the tracking error of our proposed PID control was reduced by more than $2 / 3$ compared to the case of the conventional PID control at the end of a stroke. Improving the tracking performance and considering the robustness of proposed compensation method will be studied in our future work.

\section{Acknowledgement}

This research was partially supported by Grant-in-Aid for Scientific Research (C)(19560244).

\section{References}

[1]C. Caunudas de Wit, H. Olsson, K. J. Astrom and P. Lischinsky, "A New Model for Control of System with Friction" IEEE transaction on automatic control, Vol.40, No.3, pp.419-425, 1995

[2] P.Lischinsky, C.Canudas-de-Wit, G.Morel, "Friction compensation for an Industrial Hydraulic Robot" IEEE Control Systems, Februaly, pp.25-32, 1999

[3] S.Futami, A. Furutani and S. Yoshida, "Nanometer positioning and its microdynamics"; Nanotechnology, 1, pp.31-37, 1990.

[4] J. Otsuka, T. Masuda, "The influence of nonlinear spring behavior of rolling elements on ultra-precision positioning control systems", Nanotechnology, 9, pp.85-92, 1998

[5] K.Tsuruta, T.Murakami, S.Futami, T.Sumimoto, "Genetic Algorithm (GA) Based Modeling of Non-linear Behavior of Friction of a Ball Guide Way" Proc. $6^{\text {th }}$ International Workshop on Advanced Motion Control, pp.181-186, 2000

[6] K.Tsuruta, T.Murakami, S.Futami, "Nonlinear Frictional Behavior at Low Speed in a Ball Guide Way" Proc. International Tribology Conference Nagasaki 2000, pp1847-1852, 2001

[7] K.Tsuruta, T.Murakami, S.Futami "Nonlinear Friction Behavior of Discontinuity at Stroke End in a Ball Guide Way" JSPE,Vol.69,No.12, pp.1759-1763, 2003(in Japanese) 